\title{
Model of A Critical Size Defect in the New Zealand White Rabbit's Tibia
}

\author{
Che Nor Zarida Che Seman ${ }^{1}$, Zamzuri Zakaria ${ }^{1}$, Mohd Ariff Sharifudin ${ }^{1}$, Aminudin Che Ahmad ${ }^{1}$, Mohd Shukrimi \\ Awang $^{1}$, Nazri bin Mohd Yusof ${ }^{1}$, Zunariah binti Buyong ${ }^{2}$ \\ ${ }^{1}$ Department of Orthopaedics, Traumatology \& Rehabilitation, Kulliyyah of Medicine, \\ International Islamic University Malaysia (IIUM), Jalan Hospital, 25100 Kuantan, Pahang. \\ ${ }^{2}$ Department of Basic Medical Science, Kulliyyah of Medicine, Bandar Indera Mahkota Campus, Jalan Sultan \\ Ahmad Shah, 25200 Kuantan, Pahang Darul Makmur.
}

\begin{abstract}
ABSTARCT
Introduction: Critical size defects (CSD) in the long bones of New Zealand White rabbits (Oryctolagus cuniculus) have been used for years as an experimental model for investigation of the effectiveness of a new bone substitute material. There are varieties of protocols available in the literature. This technical note attempts to present an alternative surgical technique of a CSD in the New Zealand white rabbit tibia. Methods: Thirty-nine New Zealand White rabbits were used in this study. A CSD of approximately $4.5 \mathrm{~mm}$ (width) $X 9.0 \mathrm{~mm}$ (length) was surgically drilled at the proximal tibial metaphysis, approximately $1 \mathrm{~cm}$ from the knee joint. The surrounding of soft tissue was repositioned and sutured layer by layer with bioabsorbable surgical suture. Two $\mathrm{x}$-rays of anteroposterior and lateral were taken before assessed under computed tomography scan at 6, 12 and 24 weeks. Results: This alternative method created CSD with less bleeding from the muscle observed. No mortality or other surgical complications observed within 6 weeks, 12 weeks and 24 weeks following surgery. Conclusion: A simple and safe method for performing CSD was demonstrated and recommended as an alternative approach for surgery on New Zealand White rabbits.
\end{abstract}

KEYWORDS: New Zealand White rabbit, critical size defect, surgical approach, bone healing, tibia

\section{INTRODUCTION}

Critical size defect (CSD) is an experimental approach for the in vivo assessment of tissueengineered construct. In the preclinical field of orthopaedics and trauma surgery, CSD has been used as an experimental model in evaluating the effectiveness of newly developed biomaterials to stimulate bone formation before clinical application. CSD was originally defined as "the smallest size intraosseous wound in a particular bone and species of animal that will not heal spontaneously during the lifetime of the animal" by Schmitz and Hollinger in 1986. ${ }^{1}$ However, based on American Society for Testing and Materials (ASTM) Standard Guide for Preclinical in vivo Evaluation on Critical Size Segmental Bone Defects (F2721-09), CSD model is defined as a defect that will not heal

Corresponding author:

Assoc. Prof. Dr. Zamzuri Zakaria

Department of Orthopaedics, Traumatology \&

Rehabilitation

Kulliyyah of Medicine

International Islamic University Malaysia (IIUM)

Jalan Hospital,

25100 Kuantan, Pahang.

Tel no: $+609-5706000$

Fax no: +609-5144451

Email address: zamzuri@iium.edu.my spontaneously without intervention within the lifetime of animal or experiment. ${ }^{2}$ Critical size defect model mimics the clinical situations in which substantial bone loss is present, either due to trauma or through surgery for tumour or infection.

The healing process of CSD is time consuming with new bone generation takes place slowly because of decreased blood supply to the fracture site and insufficiency of calcium and phosphorus to strengthen and harden new bone. ${ }^{3}$ Furthermore, in such defects, the migration of the fibroblasts to the wound site is faster than osteoblasts since fibrous connective tissue regenerates faster than bone tissue and become dominant in the bone tissue. ${ }^{4,5}$ In fact, the mechanism for the cellular events of wound healing at the CSD site occurred when the tissue factors such as bone morphogenetic protein or osteogenin releases from the edge of wound and caused differentiation of cells in the defect into osteoblast and chondroblasts. These cells create and mineralize the extracellular matrix forming bony islands. These islands provide scaffolding for new bone growth. However, the tissue factors are scarce in the center of the CSD, which result in a low incidence of differentiation. The chondrocytes and osteoblast fail to mineralize the matrix and eventually replaced with fibrous connective tissue. ${ }^{1}$ Therefore, repairing bone defects with biomaterials developed as defect filler, which can promote bone regeneration is essential. 
New Zealand White rabbit (Oryctolagus cuniculus) is the commonest used animal model and ranked first among all the animals used for musculoskeletal research. ${ }^{6}$ These strains of rabbit are less aggressive in nature and have less health problems as compared to other breeds. ${ }^{7}$ A healthy New Zealand White rabbit weights between 2.5 and $3.5 \mathrm{~kg}$ (semiadult) is recommended as an experimental subject because they have good capacity to withstand surgical trauma hence, preventing most serious postoperative problems and leading to a better survival rate. Gender is generally not a problem, but some researchers in the literature have preferred female sex, as it is biologically stronger. ${ }^{8}$

The rabbit model has several advantages, in terms of standardization of experimental conditions and experiment repeatability, inexpensiveness, faster skeletal change and high bone turnover rate., ${ }^{9,3}$ Wang et al. $(1998)^{10}$ have reported that there are some similarities in bone mineral density (BMD) and fracture toughness of mid-diaphyseal bone between rabbits and human being. In addition, rabbits are easily available, easy to house and handle. These characteristics make rabbits the first choice to develop an animal model for the in vivo test of a new bone substitute biomaterials. Previously, the study of bone regeneration in rabbits was performed at different anatomical sites, including the mandible $^{11}$, femur ${ }^{12,13}$, calvaria ${ }^{14}$ and radius. $^{15,16}$ It was also used in evaluating repair dynamics after having induced an injury. ${ }^{10}$

The rabbit tibia was also used as by researchers in studies involving the creation of bone defects. ${ }^{17,18,19}$ The proximal epiphysis of a rabbit tibia has a wide medial face that is devoid of muscle insertion with a surface that is slightly convex, which is suitable for induction of bone defects. Apart from the features of the anatomical site, a bone defect repair rate is mainly dependent on the bone wound size ${ }^{4}$.

A surgical technique in creating a CSD should be developed prior to testing of new biomaterials to a human being to evaluate its safety and effectiveness. Therefore, establishing an alternative surgical technique of CSD model in the New Zealand White rabbit tibia is an indispensable step for in vivo investigation of mechanical property and biocompatibility of bone substitute materials.

\section{MATERIALS \& METHODS}

This study was approved by the Institutional Animal Care and Use Committee of the International Islamic University Malaysia (IACUC-IIUM). Ethical clearance for the in vivo experimental work was obtained from the IACUC-IIUM prior to the experimental procedure (Approval Ref. No: IIUM/IACUC Approval/2016/(9)(50) dated 19 ${ }^{\text {th }}$ February 2016). Thirty-nine healthy New Zealand white rabbits weighing about 2.50 kilograms to 3.50 kilograms were used in this study. Anesthesia was administrated by a trained laboratory technician. EXPERIMENTAL PROTOCOL

\section{Anesthesia}

Preoperatively, the animals were weighted to calculate the right dosage of anesthetics, analgesia and antibiotic. The animals were anaesthetized with a mixture of $2.5 \mathrm{ml}$ Ketamine (Ketapex, Australia), $2.5 \mathrm{ml}$ Xylazine (Ilium Xylazil-100, Australia) and 250 $\mathrm{mg}$ of Tilatamine/Zolazepam (Zoletil-50, France) freeze-dried. The mixture known as KTX mixture was given at $0.2 \mathrm{ml} / \mathrm{kg}$ body weight intramuscularly for the induction and maintained at $0.1 \mathrm{ml} / \mathrm{kg}$ intravenously via marginal veins of ear during the surgery $^{20}$ (Figure 1a and Figure $1 \mathrm{~b}$ ).

\section{Preparation before surgery}

The animals were put in a supine position. The leg of the animal was shaved at the surgical site before transferring to the Animal Operating Room for surgery. Then, the leg was disinfected with providone-iodine and draped with sterile towel. All surgical procedures required the surgeon and the assistants to perform thorough surgical hand scrub and wear sterile gowns, gloves, face masks and caps to avoid infection.

\section{Surgical Approach}

The tibia tuberosity was palpated and a $3 \mathrm{~cm}$ vertical skin incision was made along the bone, exposing the subcutaneous tissue till the deep fascia (Figure 2a). The fascia was cut with sharp inside knife exposing the muscle of tibialis anterior at the upper part of the tibia. Gently the periosteal elevator was introduced to elevate and stripped the muscle of tibialis anterior on the medial side of the proximal tibial metaphysis (Figure 2b). The hemostasis was secured with a gauze and diathermy. The muscle was retracted medially using a retractor and bone spike (Figure 2c). A $2 \mathrm{~cm}$ area, exposing the proximal tibial metaphysis was created and the adequacy of the exposure was checked (Figure 2d). Using a drilling technique with a size of $4.5 \mathrm{~mm}$ drill bit, a defect was created in the area until a $4.5 \mathrm{~cm}$ (width) $\times 9 \mathrm{~mm}$ (length) defect was achieved (Figure $2 e)$. The surgical incision was closed with bioabsorbable surgical suture (Monosyn ${ }^{\circledR} 4.0$, Germany) using the continuous suturing technique for the muscle and double-layer closure with interrupted suturing techniques for skin incision (Figure $2 f$ ). Povidone soaked gauze and bandage were used for wound dressing. Figure 3 showed the CSD at the left proximal tibial of rabbit.

\section{Postoperative Care}

The animals were housed individually in cages. They were fed and watered ad libitum. There was no postoperative restriction on activity and no supportive orthotic devices used. Tramadol (5mg/ $\mathrm{kg}$ ) was given intramuscularly for pain control followed by intramuscular injection of enrofloxacin $10 \mathrm{mg} / \mathrm{kg}$ (Baytril ${ }^{\circledR} 5 \%$, Germany) as a prophylactic antibiotic daily up to seven-day post-surgery. The 
animals were monitored twice daily for a week, then daily until the time of sacrifice. The animal welfare and parameters for systemic complications such as the animal behavior, posture, weight, feeding and drinking pattern were observed and recorded.

\section{Estimated Blood Loss}

The amount of estimated blood loss per animal was measured by the number and the portion of gauze soaked with blood perioperatively. A standard size of gauze used throughout the study. Fully soaked gauze approximately equivalent to $1 \mathrm{ml}$ of blood.

\section{Radiographic Assessment}

Anteroposterior and lateral radiograph were taken at the specified period; 6 weeks, 12 weeks and 24 weeks, the radiographs were taken before assessed under computed tomography scan. The radiographs were made under consistent conditions of the tube to plate distance of $100 \mathrm{~cm}, 50$ kilovolts $(\mathrm{kV}), 3.2$ miliamperes (mAs) and 11.5 miliseconds (ms). The computed radiography systems (Phillips Optimus 80, Philips Medical System, USA) used equipped with a high-speed image reader (Regius 190, Konica Minolta) and laser printer (Drypro 793, Konica Minolta).

After that, rabbits were scanned through $360^{\circ}$ using a compact fan beam-type tomography. Data required were exported to DICOM format. Reslicing to sagittal and coronal view as well as reconstruction of 3-dimensional images was produced using OsiriX open source image processing application software for DICOM files. Evaluation of the new bone formation was based on the presence or absence of continuous bone bridging the critical size defect in any of coronal, sagittal or 3D images.

\section{RESULTS}

\section{Surgical procedure}

This approach demonstrates good exposure of the proximal tibial metaphysis to create a CSD with minimal damage to the surrounding soft tissue and less bleeding from the muscles. The number of gauze soaked with blood ranged from less than 0.5 $\mathrm{ml}$ to $4 \mathrm{ml}$ (mean: 1.12, SD: 0.59). There was no anaesthesia-related animal death recorded. The injectable anesthetic mixture dosages were appropriate for the animal and the effect lasted as long as maintained at $0.1 \mathrm{ml} / \mathrm{kg}$ intravenously via a marginal vein of the ear during the surgery. The operative time per animal ranged from 17 to 80 minutes (mean: 36.71, SD: 12.83); this duration provided ample time for induction of anesthesia, surgery, dressing of the wound, prepare the bone graft paste and subsequent transferring of the rabbits to their individual cages.

\section{Postoperative care}

All rabbits tolerated the surgery well and showed no signs of infection. The animals returned to normal activity in their cages 1 day after surgery. No signs of pain, wound infection, or abnormal behavior was detected throughout the duration of the study. There was no difficulty encountered in assessing and localizing the incision site at the shaved leg area. None of the rabbits required either a second dressing or prolonged immobilization.

\section{Radiographic Assessment}

X-ray of anteroposterior and lateral view showed radio-transparent concave of round or rectangular morphology of critical size defect. The CSD had clear and regular outlines showing a homogeneous density that clearly defined their boundaries (Figure 4a - 4f).

There was a slight reduction in the size of CSD up to 12 weeks (Figure 4b and 4e) and 24 weeks postsurgery (Figure $4 \mathrm{c}$ and $4 \mathrm{f}$ ). Furthermore, the critical size defect remained intact and not complete closure achieved by new bone formation. The medullar area's radiographic appearance did not undergo any change.

Axial view of CT scan in Figure 4 showed the margin of the defect site was blurred suggesting mineralization around the implant. There was little new bone formation from 6 weeks to 24 weeks and none of the defects bridging was observed (Figure $4 \mathrm{~g}$ $-4 i)$.
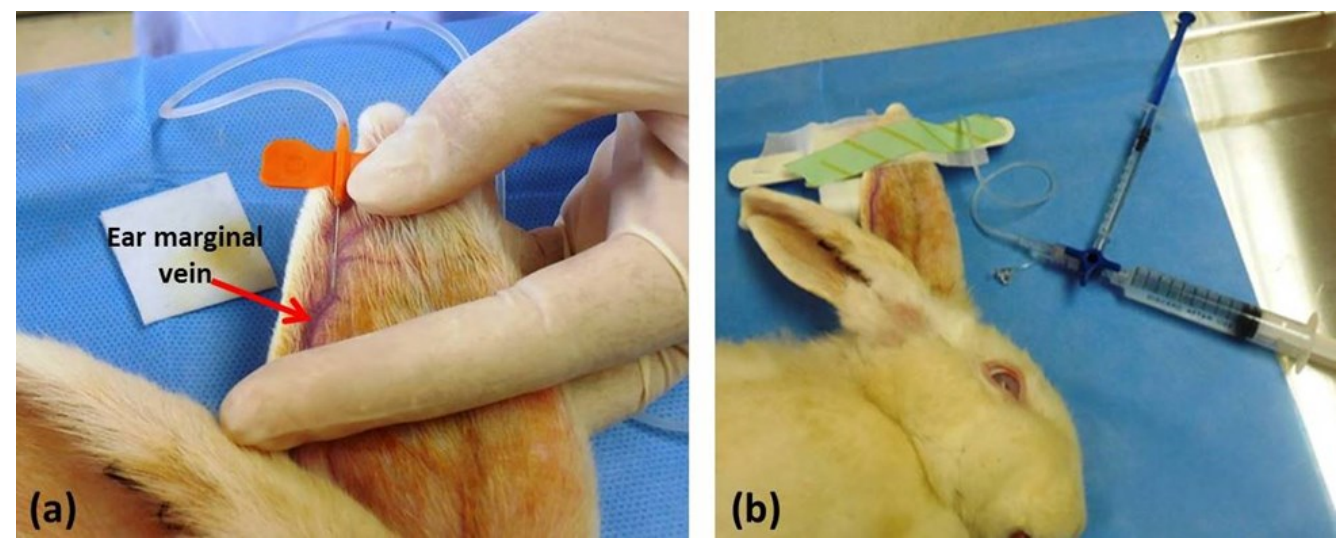

Figure 1: Animal anaesthesia. (a) Ear marginal vein (arrow). (b) The anaesthetic mixture was given by using a three-way connected to an intravenous cannula 

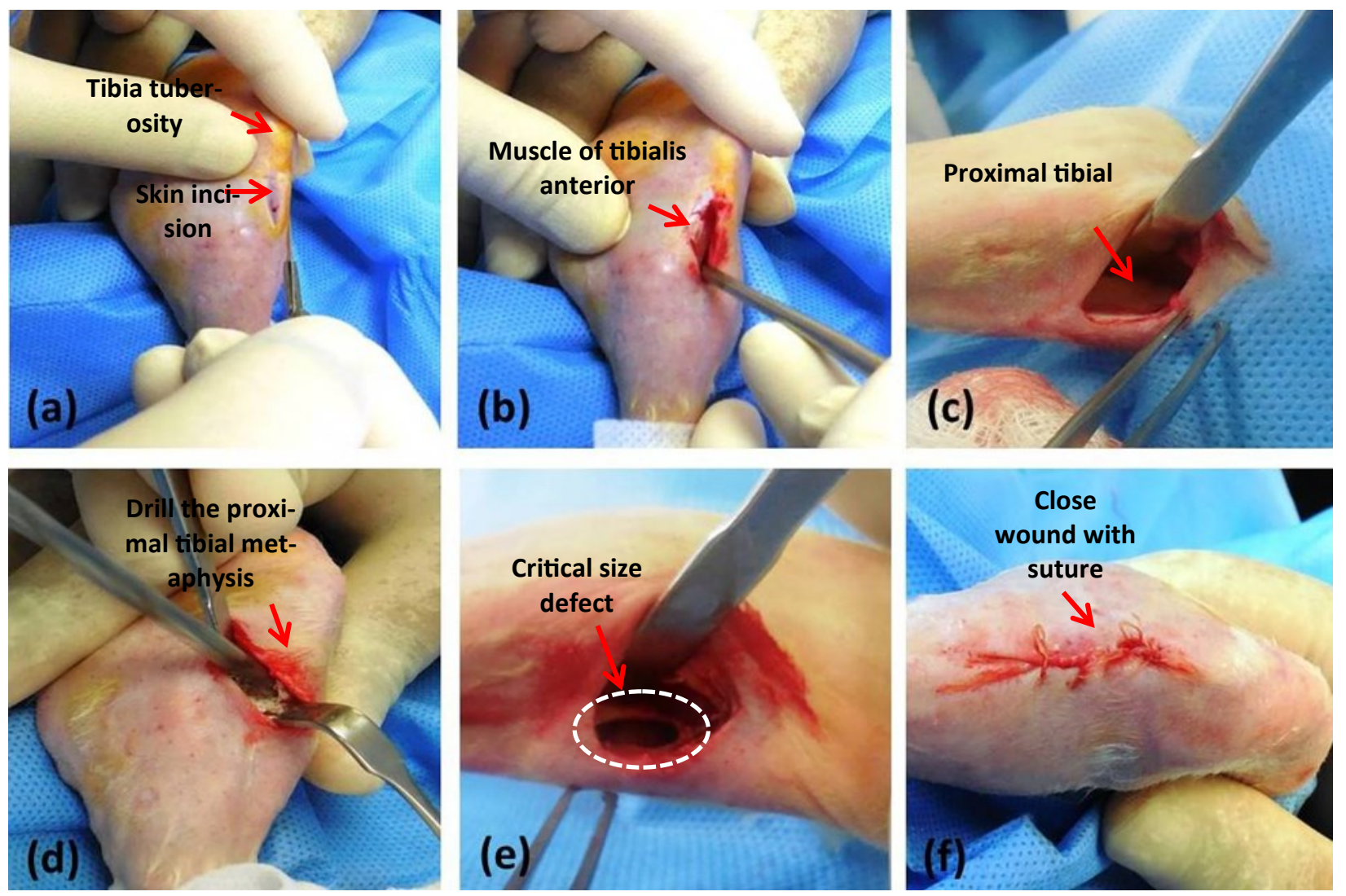

Figure 2: (a) A skin incision measuring about $3 \mathrm{~cm}$ was made at tibial tuberosity. (b) Using a periosteal elevator, the muscle was stripped from the proximal tibial metaphysis. (c) This muscle was then retracted with a retractor and bone spike exposing the bone. (d) A CSD was surgically drilled at proximal tibial metaphysis which is approximately $1 \mathrm{~cm}$ from the knee joint. (e) A CSD of approximately $4.5 \mathrm{~mm}$ (width) X $9.0 \mathrm{~mm}$ (length) (F) The wound was closed using double layer skin closure technique. Finally, povidone soaked gauze and bandage were used as a dressing.

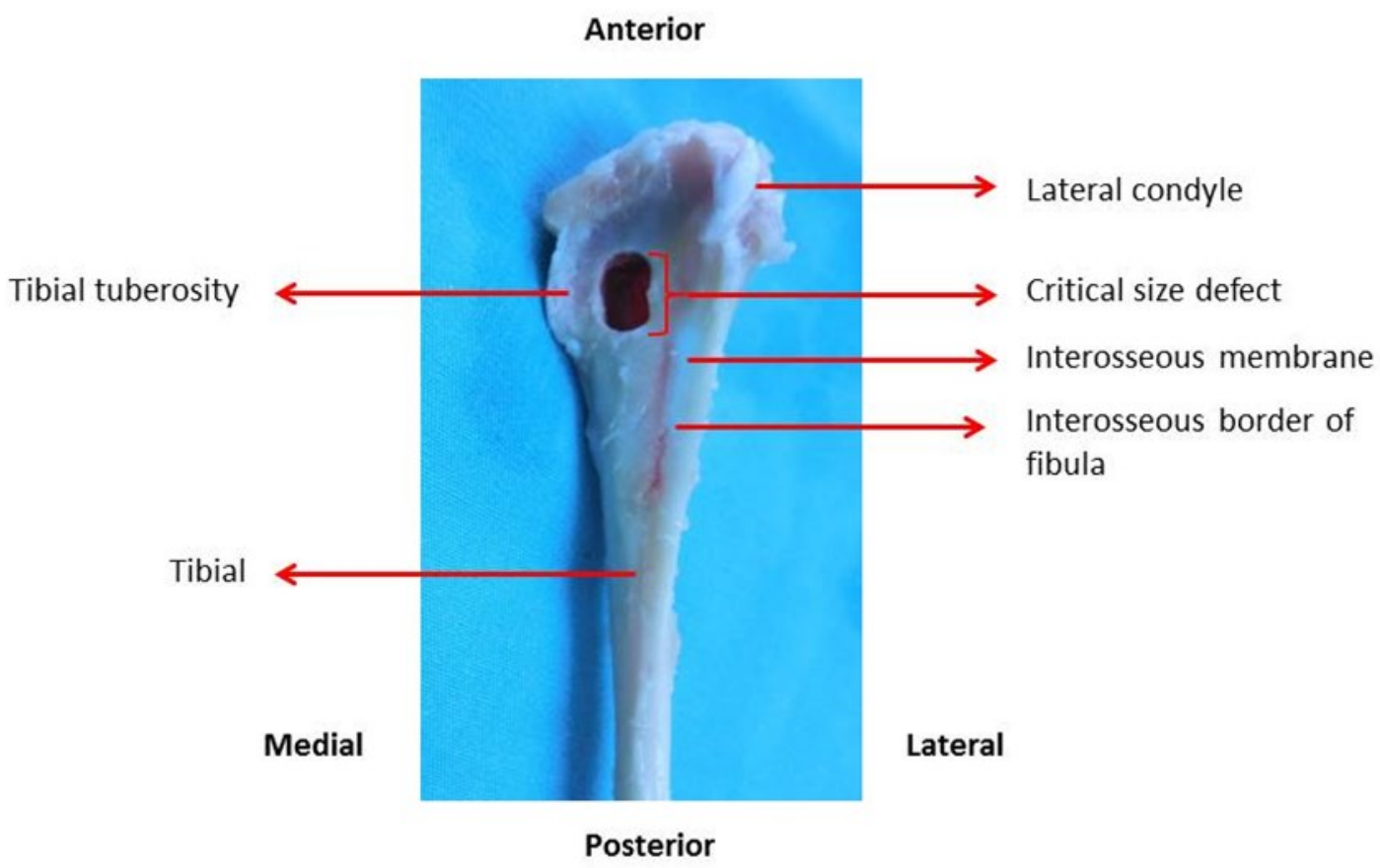

Figure 3: Critical size defect at the left proximal tibial of rabbit 

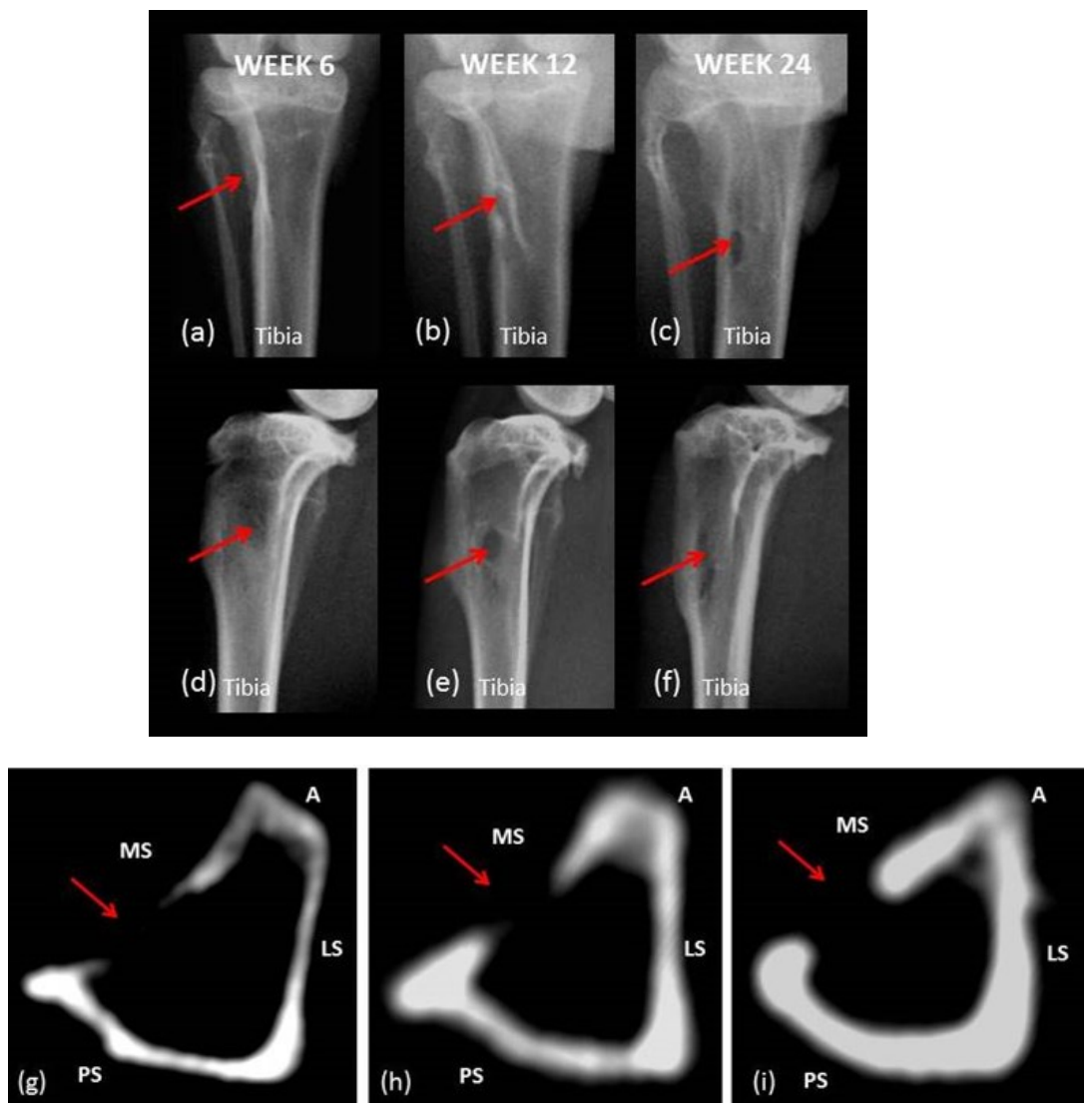

Figure 4: Radiographical observation of critical size defect; anterioposterior view at (a) 6 weeks; (b) 12 weeks; (c) 24 weeks assessment period and lateral view at (d) 6 weeks; (e) 12 weeks; (f) 24 weeks assessment period, CT scan observation of critical size defect; axial view at (g) 6 weeks, (h) 12 weeks and (i) 24 weeks assessment. Arrow indicates for the area of critical size defect; MS, medial surface; LS, lateral surface; PS, posterior surface.

\section{DISCUSSIONS}

New Zealand White Rabbit is an established animal model for evaluating the effectiveness of newly developed bone substitute materials before use for clinical application. ${ }^{3}$ The development of appropriate research protocols were reviewed by the Institutional Animal Care and Use Committee (IACUC-IIUM) involving anaesthesia procedure, surgical procedure, euthanasia procedure and carcass disposal on NZW rabbit which allowed us to carry out the research project with good quality, control and safety for both researchers and the animals.

Various sizes of CSD in rabbit tibia have been described by researchers to evaluate the biomaterials used as bone substitutes for bone defect $^{18,19,21}$ however, the size of CSD with $4.5 \mathrm{~mm}$ (width) $\times 9 \mathrm{~mm}$ (length) has never been described in details using the New Zealand white rabbit as models. Previously, Stubbs et al. (2004) had created a defect $5 \mathrm{~mm}$ wide and $15 \mathrm{~mm}$ length starting $3 \mathrm{~mm}$ below the joint line in the anteromedial cortex of the proximal tibia, CalvoGuirado et al. (2013) had created $6 \mathrm{~mm}$ diameter of CSD and Borkowski et al. (2015) had created a CSD with $4 \mathrm{~mm}$ in diameter and $6 \mathrm{~mm}$ depth about 2.5 to $3 \mathrm{~cm}$ below the epiphyseal cartilage. They found that the critical size defects were not close spontaneous. However, for the purpose of the present study, it was decided to select $4.5 \mathrm{~mm}$ width and $9 \mathrm{~mm}$ length of critical size defect. From the finding of this study showed that the critical size defects were not close spontaneously during the lifetime of the experiment and therefore, this finding proved can be used as an alternative model to create critical size defect.

The advantages of this surgical approach were less trauma since there was no nerve involved and no wound infection were observed. This finding didn't contradict with other researchers, despite they created different size of CSD. ${ }^{18,19,21}$ The amount of bleeding observed from muscle was less and all the animals survived. Our minimum blood lost during surgery was $0.5 \mathrm{ml}$, whereas the maximum was $4 \mathrm{ml}$. Blood loss during the surgery was mainly due to drilling proximal tibial metaphysis to create CSD. Compared with the previous study by Schofer et al. (2011) who created two bilateral $5 \mathrm{~mm}$ full thickness, critical size calvarial defects showed that 2 out of 30 rats died during surgery due to blood loss however no quantitative measures of blood loss was reported.

Furthermore, there was no anaesthesia-related animal death perioperatively with the used of KTX-mixture. The combination of Ketamine, Xylazine and Tilatamine/Zolazepam was administered intravenously to achieve the level of anaesthesia 
required. Based on the research procedure, the combination of drugs at the dosage of $0.1 \mathrm{ml} / \mathrm{kg}$ was safe and effective for anesthetic and analgesic plan. In order to minimize the post-surgical infection rate, the administration of antibiotic and strictly adhering to the principles of sterile surgical techniques were applied to all animals during surgery. Careful handling of the soft tissue and efficient operative technique further reduce the rate of infection. Therefore, this approach was beneficial for our study.

\section{CONCLUSION}

New Zealand White rabbit provides is an alternative model to create CSD. This alternative surgical approach provides a safe and reproducible animal model for the study of bone healing.

\section{REFERENCES}

1. Schmitz JP, Hollinger JO. The critical size defect as an experimental model for craniomandibulofacial nonunions. Clinical Orthopaedics and Related Research 1986; 205: 299-308.

2. American Society for Testing and Materials (ASTM) Standard F2721. Standard Guide for Preclinical In Vivo Evaluation in Critical Size Segmental Bone Defects, ASTM International,West Conshohocken, Pa, USA, 2008.

3. Li Y, Chen S, Li L, Qin L. Bone defect animal models for testing efficacy of bone substitute biomaterials. Journal of Orthopaedic Translation 2015; 3: 95-104. Available at: http:// doi.org/10.1016/j.jot.2015.05.002. Accessed July 28, 2016.

4. Liu $Y$, Wu G, Groot K et al. Biomimetic coatings for bone tissue engineering of critical-sized defects. J. R. Soc. Interface 2010; 1-17. Available at http:// rsif.royalsocietypublishing.org/. Accessed July 28, 2016.

5. Stetzer K, Cooper G, Gassner R et al. Effects of fixation type and guided tissue regeneration on maxillary osteotomy healing in rabbits. Journal of Oral Maxillofacial Surgery 2002; 60:436-437.

6. Neyt J, Buckwalter JA, Carroll N. Use of animal models in musculoskeletal research. lowa Orthop J 1998; 18:118-123.

7. Suckow MA, Douglas FA. The laboratory Rabbit. New York: CRC Press, Inc., 1997.

8. Mapara M, Thomas BS, Bhat KM. Rabbit as an animal model for experimental research. Dental Research Journal [serial online] 2012; 9(1), 111118. Available at: http://doi.org/10.4103/17353327.92960. Accessed 15 June, 2016.

9. Castaneda S, Largo R, Calvo E. Bone mineral measurements of subchondral and trabecular bone [serial online] in healthy and osteoporotic rabbits. Skeletal Radiology 2006; 35(1): 34-41. Available at: http://doi.org/10.1007/s00256-0050022-z. Accessed July 12, 2016.

10. Wang X, Mabrey JD, Agrawal CM. An interspecies comparison of bone fracture properties. Biomedical Material Engineering 1998; 8: 1-9.

11. Zhang JC, Lu HY, Lu HY. et al. The repair of critical-size defects with porous hydroxyapatite/polyamide nanocomposite: an experimental study in rabbit mandibles. International Journal of Oral and Maxillofacial Surgery 2010; 39: 469-477.

12. Wheeler DL, Eschbach EJ, Hoellrich RG. et al. Assessment of resorbable bioactive material for grafting of critical-size cancellous defects. Journal of Orthopaedic Research 2000; 18: 140-148.

13. Peterson B, Zhang J, Iglesias R. et al. Healing of Critically Sized Femoral Defects, Using Genetically Modified Mesenchymal Stem Cells from Human Adipose Tissue. Tissue Engineering 2005; 11(1-2): 120-129.

14. Cowan CM, Shi YY, Aalami OO. et al. Adipose -derived adult stromal cells heal critical-size mouse calvarial defects. Nature Biotechnology 2004; 22(5): 560-567.

15. Saito A, Suzuki Y, Kitamura M. et al. Repair of 20-mm long rabbit radial bone defects using BMP-derived peptide combined with an a-tricalcium phosphate scaffold. Journal of Biomedical Materials Research Part A 2006; 77A (4): 700-706.

16. Kasten P, Vogel J, Geiger F. et al. The effect of platelet-rich plasma on healing in criticalsize long-bone defects. Biomaterial 2008; 29: 3983-3992.

17. Russell N, Oliver, RA, Walsh WR. The effect of sterilization methods on the osteoconductivity of allograft bone in a critical-sized bilateral tibial defect model in rabbits. Biomaterials 2013; 34(33): 81858194.

18. Calvo-Guirado JL, Ramirez-Fernandez MP, Delgado-Ruiz RA. et al. Influence of Biphasic $B$-TCP with and without the use of collagen membranes on bone healing of surgically critical size defects. A radiological, histological, and histomorphometric study. Clinical Oral Implants Research 2014; 25(11): 1228-1238.

19. Borkowski L, Pawlowska M, Radzki RP. Effect of a carbonated HAP/B-glucan composite bone substitute on healing of drilled bone voids in the proximal tibial metaphysis of rabbits. Materials Science and Engineering $C$ 2015; 53: 60-67.

20. Cheah PS, Low CK, Mohidin N. et al. Ketamine-xylazine/tiletamine-zolazepam prolonged anesthesia in Cyanomolgus monkeys. Online Journal of Veterinary Research 2007; 11(1): 39-45.

21. Stubbs, D., Deakin, M., Chapman-Shealth, P. et al. In vivo evaluation of resorbable bone graft substitutes in a rabbit tibial defect model. Biomaterials 2004; 23: 5037-5044 